\title{
Tecnologias de Internet em uma plataforma de colaboração para a pesquisa médica
}

\author{
DOI: $10.3395 /$ reciis.v3i4.236pt
}

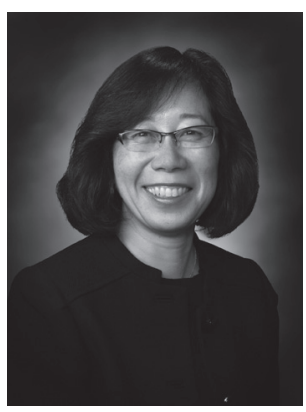

Miriam Akemi

M.Capretz

Departamento de

Engenharia Elétrica e de

Computação, University

of Western Ontario,

Ontario, Canada

mcapretz@eng.uwo.ca

\section{Marcelo Fantinato}

Universidade de São Paulo, São Paulo, Brazil m.fantinato@usp.br

\section{Shuying Wang}

University of Western Ontario, Ontario, Canada swang259@uwo.ca

\section{Olga Nabuco}

Centro de Tecnologia da Informação Renato Archer-CTI, Campinas, Brazil

olga.nabuco@cti.gov.br

\section{Rodrigo Bonacin}

Centro de Tecnologia da Informação Renato Archer-CTI, Campinas, Brazil rodrigo.bonacin@cti.gov.br

\section{Itana Gimenes}

Universidade Estadual de Maringá, Maringá, Brazil

itana@din.uem.br

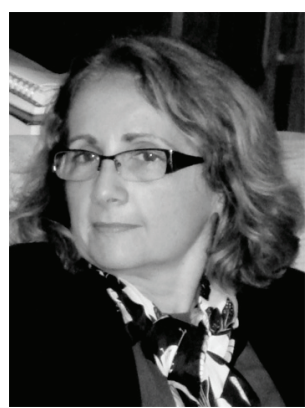

Maria

Beatriz Felgar Toledo

Instituto de

Computação, Unicamp,

Campinas, Brazil

beatriz@ic.unicamp.br

\section{Diego Zuquim G. Garcia}

Unicamp, Campinas, Brazil

diego.garcia@ic.unicamp.br

\section{David Allison}

University of Western Ontario, Ontario, Canada dallison@uwo.ca

\section{Marcos Rodrigues}

Centro de Tecnologia da Informação Renato Archer-CTI, Campinas, Brazil marcos.rodrigues@cti.gov.br

\section{Emma Chen Sasse \\ Unicamp, Campinas, Brazil \\ emma.sasse@uol.com.br}

\section{Americo Brigido Cunha Centro Federal de Educação Tecnológica do Rio de Janeiro-CEFET, Rio de Janeiro, Brazil americobc@gmail.com}




\section{Resumo}

As tecnologias de Internet mudaram o desenvolvimento de software. As mudanças afetam uma gama completa de aplicações, assim como a forma em que os usuários interagem com os computadores. No campo da saúde, a pesquisa médica demanda muito investimento, esforço e informações para a comercialização segura de um fármaco novo. O projeto WebInVivo visa fornecer suporte automatizado à pesquisa médica, alicerçado em tecnologias de Internet. Ele inclui mecanismos para o compartilhamento e a reutilização de informações de pesquisas médicas, tais como protocolos, dados de protocolos, fluxos de trabalho e metadados de fluxos de trabalho para o controle do ciclo de vida do protocolo, da modelagem à execução. Neste projeto, o conhecimento da área biomédica permeia três segmentos da sociedade brasileira: (a) pesquisa e desenvolvimento, (b) agentes de saúde e (c) a população. Este conhecimento será disponibilizado em redes sociais, para esses segmentos da sociedade brasileira.

\section{Palavras-chave}

tecnologias de internet; computação orientada a serviços; gestão do fluxo de trabalho; redes sociais

\section{Introdução}

A computação orientada a serviços (PAPAZOGLOU e VAN DEN HEUVEL, 2007) e sua aplicação em tecnologias de serviços da Web (ALONSO et al., 2004) mudou a forma como os sistemas de software são estruturados e desenvolvidos. Uma gama completa de aplicações em negócios, administração pública, saúde, educação, cultura e entretenimento pode beneficiar-se das tecnologias de Internet, como o reuso de funcionalidades, a redução de custo e a eficiência operacional. Ademais, as tecnologias de serviços de Web também são amplamente adotadas devido à disponibilidade de muitos padrões, por exemplo a Web Services Description Language WSDL (WSDL, 2007) e a Web Services Business Process Execution Language - WS-BPEL (ALVES et al., 2007).

As tecnologias Web 2.0 (O’REILLY, 2005), incluindo as redes sociais e wikis, aumentaram a interatividade e o conteúdo gerado por usuários, encontrado na Internet original (BERNERS-LEE, 1999), o que promove novos tipos de aplicações e simplifica a forma como os usuários interagem com os computadores.

A Internet Semântica (BERNERS-LEE et al., 2001) permite que a Internet seja usada por programas de computador e também por pessoas, através da formalização do conhecimento, conhecida como ontologia (GRUBER, 2007). Ela pode ser entendida como um modelo conceitual que apresenta uma formalização lógica que descreve a palavra com rigor. Esse modelo permite que a Internet seja usada não apenas como um conjunto de documentos interrelacionados por hiperlinks, mas também como uma rede semântica de conceitos.

Noâmbitodasaúde, abaixaprevisibilidadedos processos e o acoplamento pouco rígido das unidades organizacionais respaldam o argumento do potencial da plataforma de colaboração nas organizações. A previsibilidade dos processos na pesquisa médica pode ser classificada como não estruturada, ad hoc ou com estrutura livre, tal como definido por Dumas, Aalst e Hofstede (2005). Os processos são muito diferentes da abordagem dos processos de negócios, em que a estrutura das atividades é rígida - o que é comum em organizações com fins lucrativos. Outra característica importante das organizações de saúde é o fato de trazerem em si "um modelo modular, com acoplamento pouco rígido, no qual unidades separadas e semiautônomas se especializam em áreas distintas de prestação de cuidados à saúde" (PINELLE e GUTWIN, 2006). A relação de acoplamento pouco rígido entre as unidades acarreta um fluxo limitado de informações, tanto através da organização quanto entre os grupos de pesquisa. O grau de entendimento e responsabilidade no controle e no planejamento das atividades é aumentado pela visibilidade disponível na plataforma de colaboração (DUMAS et al., 2005). Organizações de renome, como a Organização para Cooperação e Desenvolvimento Econômico (OCDE) e o Sétimo Programa-Quadro da União Europeia, são unânimes ao propor soluções que enfatizam a necessidade de gestão e compartilhamento do conhecimento gerado no curso da Pesquisa e do Desenvolvimento (P\&D) da nova medicina (CALLAN e GILLESPIE, 2007; IMI, 2008). Essas organizações sugerem a criação de uma rede virtual global com uma arquitetura de informações sustentável para o compartilhamento e o intercâmbio de informações, dados e ferramentas de pesquisa que, ao mesmo tempo, salvaguarde a propriedade intelectual e os objetos da pesquisa.

$\mathrm{O}$ projeto WebInVivo enfoca a pesquisa médica e as etapas da mesma para o desenvolvimento 
de fármacos novos. Considerando que a pesquisa médica é um processo caro, de longa duração, as ferramentas do software WebInVivo foram propostas para otimizar o uso de recursos, minimizar os esforços na pesquisa de fármacos novos, simplificar o fluxo de informações entre os pesquisadores e reduzir o tempo até a comercialização do fármaco.

O projeto WebInVivo usa tecnologias de serviços Web e ferramentas de colaboração Web 2.0 para atender as necessidades da pesquisa médica, especificamente a pesquisa de apoio ao compartilhamento do conhecimento durante as experiências. Nas ciências biomédicas, o conhecimento permeia três grupos da sociedade brasileira, com necessidades e características distintas. Em primeiro lugar, afeta as equipes de P\&D que trabalham na descoberta e no desenvolvimento de medicações e tratamentos novos. Em segundo lugar, envolve a equipe de profissionais de saúde, como médicos, enfermeiras, administradores, técnicos e educadores. Por fim, impacta a população exposta a doenças e que não dispõe de informações sobre elas. Neste projeto, o conhecimento estará disponível aos três segmentos da sociedade mencionados, através de redes sociais, de acordo com as necessidades dos mesmos e com o grau de instrução. Este desafio cria iniciativas novas nas áreas de P\&D de saúde e gestão de conhecimento, no que tange a diversidade de usuários envolvidos no processo de produção e consumo de informações.

Alguns projetos, como o MyExperiment (ROURE et al., 2008), oferecem suporte a experiências que usam a arquitetura Web 2.0, em escopos que vão além da pesquisa médica. Arden (CLAYTON et al., 1989), GLIF (PELEG et al., 2004) e Gaston (CLERCQ \& HASMAN, 2004) têm como meta todas as aplicações médicas, mas não se fundamentam no paradigma orientado a serviços, nem nos recursos da Web 2.0 para colaboração e compartilhamento. Propõem uma arquitetura em camadas, em que cada camada envolve um conjunto de componentes. Alguns componentes foram implementados; maiores detalhes podem ser encontrados nos ensaios mencionados neste artigo.
No todo, o artigo propõe uma arquitetura embasada em tecnologias de Internet que oferecem apoio à pesquisa médica. $\mathrm{O}$ restante deste artigo está estruturado desta forma: A Seção 2 apresenta os conceitos básicos relacionados ao projeto, enquanto a arquitetura WebInVivo é discutida na Seção 3. O trabalho e os benefícios correlatos de uma arquitetura fundamentada na Internet são apresentados nas seções seguintes. Por fim, as descobertas e o estado atual do projeto concluem o artigo.

\section{Fundamentação}

A pesquisa médica é uma etapa crítica no desenvolvimento de fármacos e, por isso, requer apoio para o compartilhamento e a reutilização do conhecimento para a aceleração do progresso das experiências e a otimização dos recursos. A plataforma WebInVivo foi proposta para atender essas necessidades; ela fornece a tradução de procedimentos de pesquisa clínica em fluxos de trabalho científico, além do compartilhamento de informações através de redes sociais. Os conceitos básicos desta plataforma serão descritos nesta seção.

\section{Pesquisa médica}

$\mathrm{O}$ processo de desenvolvimento de um fármaco, retratado na Figura 1, consiste em várias fases. Uma dessas fases é a pesquisa médica, que contém uma sequência rigorosa de etapas que devem ser aferidas e registradas. A Fase 1 avalia os efeitos dos fármacos novos em voluntários saudáveis. $\mathrm{Na}$ Fase 2, a segurança/ eficácia do fármaco é testada em uma população de pacientes. Isso pode durar até dois anos. Nessa fase são produzidas informações de comparação quanto à segurança do fármaco novo e sua eficácia. Apenas cerca de um terço dos fármacos experimentais concluem com êxito a Fase 2. Na Fase 3, a pesquisa envolve uma maior população de teste de pacientes, com o fármaco novo, em comparação com a terapia padrão ou placebo. Esta fase fornece um entendimento

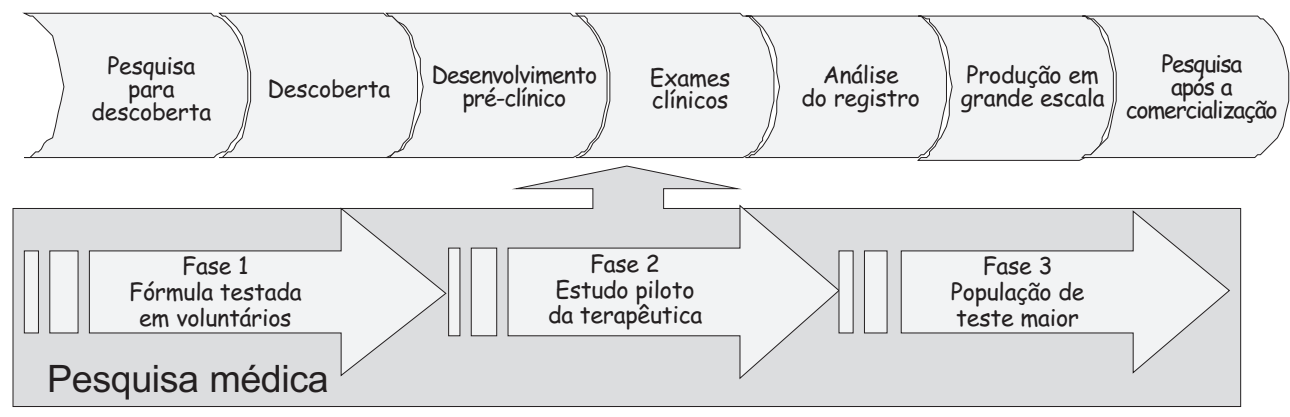

Figura 1 - Fases da pesquisa médica 
mais abrangente sobre a eficácia do fármaco e as reações adversas possíveis. A Fase 3 pode durar vários anos. De setenta a noventa por cento dos fármacos que alcançam esta fase a concluem com êxito. Dependendo dos resultados, o fármaco poderá ser comercializado ou não.

As fases da pesquisa médica têm de ser descritas com clareza, apresentar rigor científico, ser passíveis de verificação, e todas as alterações têm de ser relatadas. Para garantir que essas exigências sejam atendidas, um protocolo deve ser elaborado, aprovado e registrado antes do início da pesquisa. Uma pesquisa médica envolve coleta de informações, registro da evolução do tratamento e análise de dados. O processo como um todo pode beneficiar-se do desenvolvimento e do uso de novas ferramentas de software para a entrada de dados, coleta de dados, acompanhamento dos procedimentos e visualização dos resultados.

A solução antevista engloba a automação da execução do protocolo e o suporte à colaboração entre as possíveis partes interessadas, incluindo o governo, entidades normativas, pesquisadores, agentes de saúde e cidadãos. A colaboração é fundamental para congregar esforços, acelerar os processos de pesquisa e otimizar os recursos disponíveis. As novas ferramentas de software garantem um nível maior de confiabilidade dos dados e oferecem apoio mais sólido à execução do protocolo.

\section{Protocolos clínicos e fluxos de trabalho}

Um protocolo clínico é um plano de estudo em que se fundamentam todas as pesquisas médicas. O plano deve ser projetado com cuidado para salvaguardar a saúde dos participantes e também para responder perguntas de pesquisa específicas. O protocolo tem de descrever as funções dos participantes em uma pesquisa específica, o cronograma de testes, os procedimentos, medicações e dosagens, além da duração do estudo. Em uma pesquisa médica, os participantes que cumprem um protocolo têm consultas regulares com a equipe de pesquisa para a monitoração da sua saúde e para a determinação da segurança e eficácia do tratamento. Além disso, todas as visitas e procedimentos têm de ser agendados com antecedência. Atualmente, o protocolo é redigido como um formulário, mas ele pode ser elaborado como um fluxo de trabalho para oferecer um panorama dos procedimentos envolvidos na pesquisa.

O fluxo de trabalho descreve um conjunto de atividades projetadas para alcançar uma meta organizacional. Ele contém uma descrição precisa da sequência de atividades que tem de ser seguida para alcançar a meta determinada. A implementação das atividades do fluxo de trabalho envolve tanto pessoas quando sistemas computacionais. Muitas ferramentas já foram desenvolvidas para apoiar a gestão do fluxo de trabalho; elas podem ser adaptadas e reutilizadas na área específica da pesquisa médica.

O Quadro 1 apresenta um exemplo de protocolo para o estudo de um fármaco específico. Segundo este protocolo, há procedimentos diferentes que podem ser aplicados às diversas fases do estudo, alguns dos quais se repetem em mais de uma fase. Esses procedimentos podem envolver

Quadro 1 - Fluxo de trabalho

\begin{tabular}{|c|c|c|c|c|c|c|c|c|}
\hline Fases do Estudo & Triagem & & oses de & "ataqu & & Doses $\mathrm{me}$ & ensais & Fim do estudo \\
\hline Visitas & Triagem & $\begin{array}{l}\text { Inclusão } \\
+1^{\text {a }} \\
\text { Dose }\end{array}$ & $\begin{array}{c}2^{a} \\
\text { dose }\end{array}$ & $\begin{array}{c}3^{a} \\
\text { dose }\end{array}$ & $\begin{array}{c}\text { Avaliação } \\
\text { da } \\
\text { resposta }\end{array}$ & $\begin{array}{l}\text { Meses } \\
\text { pares }\end{array}$ & $\begin{array}{c}\text { Meses } \\
\text { ímpares }\end{array}$ & $\begin{array}{c}\text { Avaliação da } \\
\text { resposta }\end{array}$ \\
\hline Procedimenta & $\begin{array}{l}14 \text { dias } \\
\text { antes do } \\
\text { início }\end{array}$ & Início & \begin{tabular}{|c|}
1 dia \\
após o \\
início
\end{tabular} & $\begin{array}{l}1 \text { dia } \\
\text { após o } \\
\text { início }\end{array}$ & $\begin{array}{c}5 \text { a } 8 \text { dias } \\
\text { após o } \\
\text { início }\end{array}$ & $\begin{array}{c}\text { Meses após } \\
\text { o início }\end{array}$ & $\begin{array}{l}\text { Meses } \\
\text { após o } \\
\text { início }\end{array}$ & $\begin{array}{c}30 \text { dias após a } \\
\text { última dose }\end{array}$ \\
\hline Qualificação & $\mathrm{X}$ & $x$ & & & & & & \\
\hline $\begin{array}{l}\text { Exame físico e } \\
\text { histórico }\end{array}$ & $x$ & & & & & $x$ & $x$ & $x$ \\
\hline $\begin{array}{c}\text { Medicação } \\
\text { concomitante }\end{array}$ & $x$ & $x$ & $x$ & $x$ & $x$ & $x$ & $x$ & $x$ \\
\hline $\begin{array}{c}\text { Consentimento } \\
\text { informado }\end{array}$ & $x$ & & & & & & & \\
\hline Avaliação de dor & & $x$ & & & $x$ & $x$ & $x$ & $x$ \\
\hline $\begin{array}{c}\text { Tabela de } \\
\text { qualidade de vida }\end{array}$ & & $x$ & & & & $x$ & & $x$ \\
\hline Toxicidade & & $x$ & $X$ & $x$ & $x$ & $x$ & $x$ & $x$ \\
\hline $\begin{array}{c}\text { Infusão de fármaco } \\
\text { experimental }\end{array}$ & & $x$ & $x$ & $x$ & & $x$ & $x$ & \\
\hline
\end{tabular}


apenas o pesquisador, ou também podem envolver os pacientes que participam do estudo, o que requer o intercâmbio de informações entre várias partes.

As duas fases iniciais do Quadro 1 foram definidas como um fluxo de trabalho, com o uso da Business Process Modeling Notation (BPMN), nas Figuras 2(a) e 2(b). A primeira fase representa a fase de 'triagem', enquanto a segunda fase foi denominada fase de 'inclusão mais primeira dose'. Em cada fase, tarefas, pontos de acesso e fluxos de sequência são modelados para todas as funções do pesquisador e dos participantes, bem como o fluxo de mensagens entre eles.

Recentemente, a tecnologia de fluxo de trabalho passou a atrair muito o interesse da comunidade científica, principalmente no caso dos sistemas de fluxo de trabalho científico (TAYLOR et al., 2006). Esses sistemas permitem o compartilhamento e a cooperação entre os cientistas, no desenvolvimento de experiências científicas (ROURE et al., 2008). Os Sistemas de Gestão do Fluxo de Trabalho (WfMS - sigla de Workflow Management Systems) fornecem suporte automatizado ao projeto, execução e monitoramento do fluxo de trabalho. Além disso, interpretam as definições dos processos, interagem com as pessoas participantes e recorrem a ferramentas e aplicações para a realização das atividades do fluxo de trabalho.

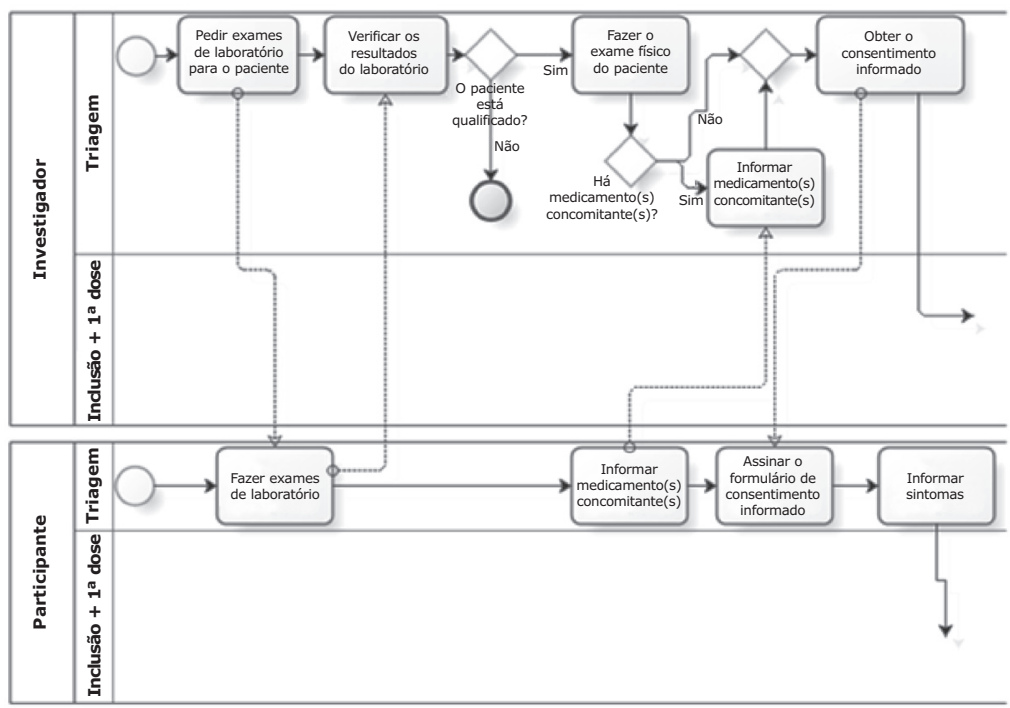

Figura 2 (a) - O protocolo como fluxo de trabalho em BPMN

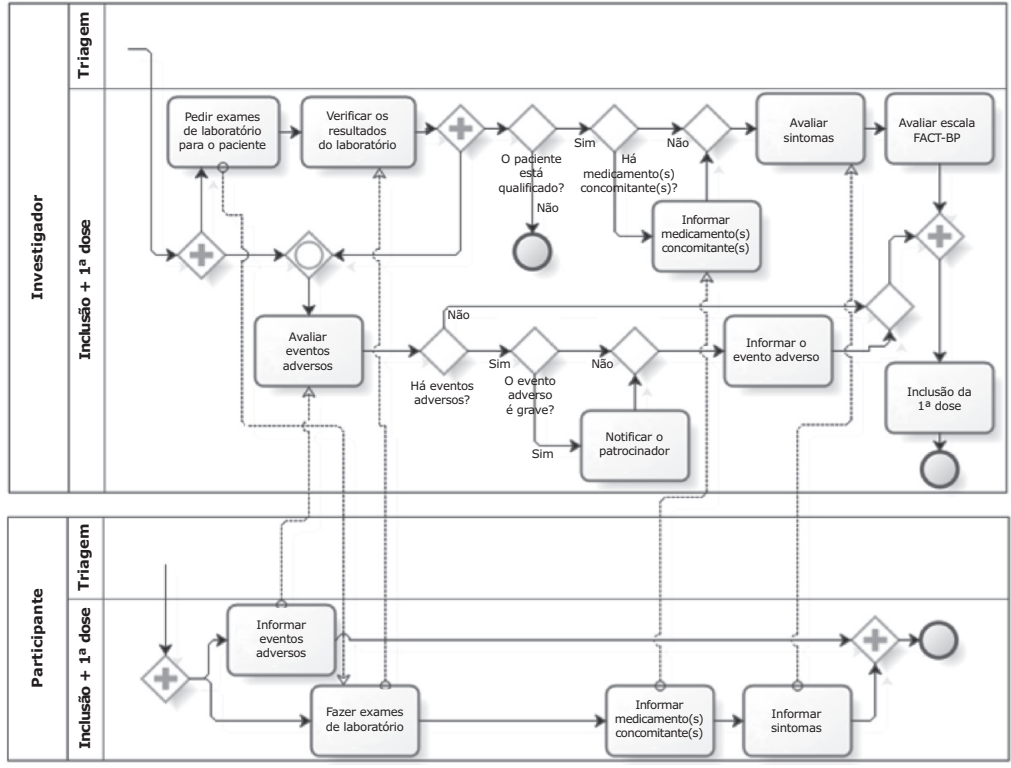

Figura 2 (b) - O protocolo como fluxo de trabalho em BPMN 


\section{Web 2.0 e Redes Sociais}

A concepção original da Internet, tal como proposta por Berners-Lee (1999), permite a interação humana com uma biblioteca de documentos interconectados por links de hipermídia. Programas de computador como os navegadores apresentam esses documentos, denominados páginas da Internet, enquanto as pessoas são responsáveis pelo encadeamento das páginas da Internet e pela interpretação do seu conteúdo. Em contraposição a essa visão inicial, a Internet Semântica (BERNERS-LEE et al., 2001) oferece suporte à análise por máquina e à integração entre programas. Esse modelo permite o uso da Internet não apenas como um conjunto de documentos inter-relacionados por hiperlinks, mas também como uma rede semântica de conceitos.

As tecnologias Web 2.0 (O’REILLY, 2005), como blogs, fóruns de discussão e wikis aumentam a interatividade e o conteúdo gerado pelos usuários em comparação com a Internet original. Os blogs permitem que as pessoas elaborem diários com itens aos quais os leitores podem adicionar comentários, em ordem cronológica inversa. Os fóruns de discussão oferecem oportunidades para debates democráticos sobre temas variados. Além disso, as redes sociais permitem que as pessoas participem de grupos com interesses semelhantes, com os quais podem interagir on-line e compartilhar mídias. Por fim, os wikis permitem que os usuários gerem conteúdo, de forma conjunta, através da atualização de páginas da Internet.
Uma rede social consiste em nodos, que representam pessoas ou organizações, interconectados por um ou mais tipos de relação, como amizade, interesses ou preferências. A rede é responsável pelo compartilhamento de ideias entre pessoas com interesses, objetivos e valores comuns. No contexto médico, também há exemplos de redes sociais em escopos fora da pesquisa médica, como a PatientsLikeMe (http://www.patientslikeme. com), usada para compartilhar informações sobre doenças. O DailyStrength (http://dailystrength. org) é um site para apoio emocional, e o Sermo (http://www.sermo.com) é um fórum on-line para o intercâmbio de opiniões profissionais de médicos.

\section{A arquitetura WebInVivo}

A arquitetura WebInVivo oferece suporte a um ambiente de pesquisa de colaboração, estruturado em quatro camadas, tal como apresentado na Figura 3. Essas camadas são:

-Redes Sociais: conectam os três grupos da sociedade brasileira, alvo deste projeto.

-Interface Pessoa-Computador: fornece adaptação conforme o uso e as necessidades de categorias de usuários específicas.

- Lógica da Aplicação: consiste nos componentes da gestão de serviço, fluxo de trabalho e protocolo.

- Conteúdo e Recursos: inclui wikis, protocolos, exemplos de fluxo de trabalho e ontologias.

As subseções acima descrevem cada uma dessas camadas.

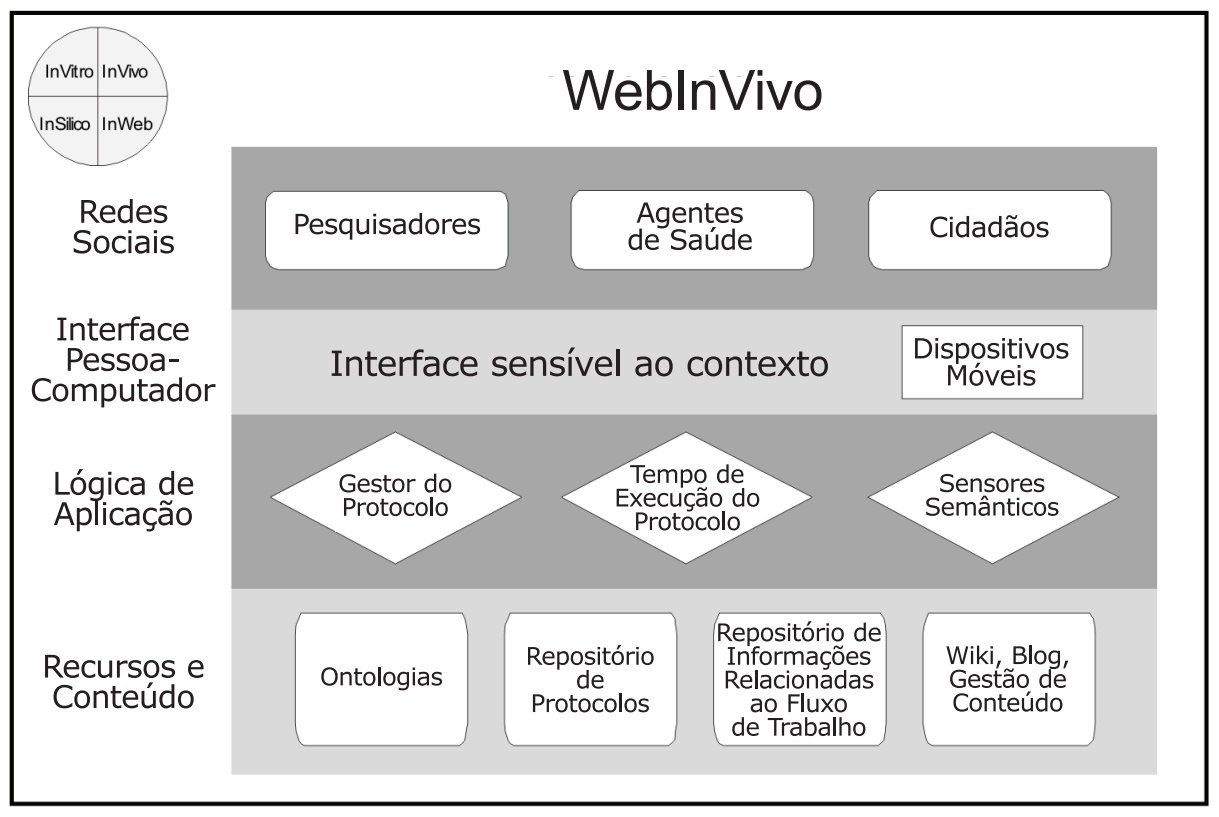

Figura 3 - arquitetura WebInVivo 


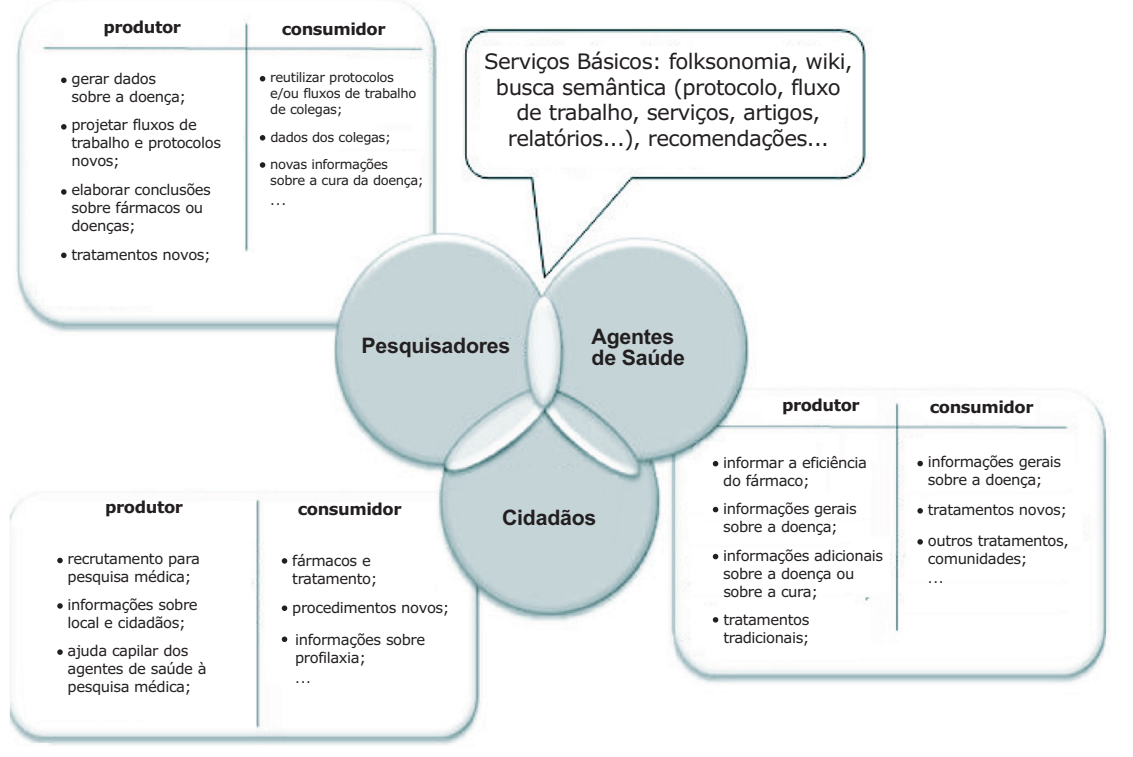

Figura 4 - as três redes sociais

\section{A camada de redes sociais}

A camada de Redes Sociais, apresentada na Figura 4, simplifica o trabalho de três grupos da sociedade brasileira e inclui: a Rede dos Pesquisadores, a Rede dos Agentes de Saúde e a Rede dos Cidadãos. O conceito da "Cauda Longa" (O'REILLY, 2005; ROURE et al., 2008) pode ser aplicado a essas redes, uma vez que os ativos digitais da pesquisa médica, que incluem protocolos, fluxos de trabalho, serviços na Internet, artigos e vídeos de treinamento, serão desenvolvidos e compartilhados entre pesquisadores e profissionais de saúde geograficamente separados. Eles usarão os resultados como procedimentos para prevenção, diagnóstico e tratamento. O conceito de "efeitos da rede" (O’REILLY, 2005; ROURE et al., 2008) também pode ser aplicado quando os participantes da Rede dos Pesquisadores usarem os ativos digitais e adicionarem dados, tais como os efeitos colaterais do uso. Rótulos são usados para as associações, o que permite a criação de conhecimento com base no uso. A Rede dos Cidadãos deve ter cobertura nacional das informações acessíveis para prevenção e tratamento, com múltiplos canais de acesso, incluindo TV digital e dispositivos móveis (SORIANO et al., 2008).

A Rede dos Pesquisadores será fundamentada na tecnologia de Internet Semântica. Ela permitirá o compartilhamento do protocolo, compartilhamento do fluxo de trabalho, execução do fluxo de trabalho, compartilhamento de dados, armazenamento de recursos e pesquisa. Além disso, a Rede dos Agentes de Saúde terá acesso parcial aos resultados de tratamento e prevenção. Também poderá contribuir para o projeto do fluxo de trabalho e a revisão do protocolo, através da inclusão de comentários nos wikis correspondentes. Por fim, a Rede dos Cidadãos terá acesso a informações sobre prevenção e tratamento. Ela também poderá participar diretamente das pesquisas, à medida que os agentes dos fluxos de trabalho respondam aos questionários e, no futuro, interagir através de sensores específicos.

As interconexões nas redes sociais contam com o apoio da nossa arquitetura proposta, fundamentada em nosso padrão orientado aos serviços. Os serviços que oferecem suporte à folksonomia, buscas semânticas, fluxos de trabalho, serviços, artigos e dados permeiam as redes, fornecendo o recurso de compartilhar o conhecimento gerado. Essas redes ficarão interconectadas, gerando eventos e alertas sobre situações de crise: epidemias, condições meteorológicas, infestações por vetores (dengue, malária, etc.). Os tratamentos novos e os fármacos novos também podem fazer parte desses eventos. Os efeitos colaterais podem ser informados e alcançar a comunidade específica relacionada aos mesmos.

Os ativos produzidos e consumidos por pesquisadores, agentes de saúde e cidadãos foram destacados nos retângulos arredondados da Figura 4. Mais especificamente, os pesquisadores apresentam dados sobre doenças, além de projetar e desenvolver protocolos, fluxos de trabalho, serviços na Internet e bancos de dados de pesquisa médica; 
por outro lado, podem consumir os ativos de outros pesquisadores. Os agentes de saúde colaboram com as pesquisas médicas e busca de informações sobre tratamentos, procedimentos e fármacos novos. Os cidadãos também colaboram com as pesquisas médicas, apresentando informações complementares, além de pesquisar informações gerais sobre as doenças.

As atividades relacionadas à produção e ao consumo de recursos contam com o apoio da arquitetura WebInVivo. Elas incluem o projeto e o tempo de execução dos serviços de suporte à pesquisa médica, protocolo e ferramentas de projeto do fluxo de trabalho, gestão do processo de pesquisa médica, e serviços de apoio à gestão do conhecimento, como sistemas de recomendação, folksonomia e busca semântica.

\section{A camada de interface pessoa- computador}

As redes sociais propostas incluem usuários com vários níveis de instrução e linguagens, que podem vivenciar condições ambientais distintas e/ou ter métodos de acesso diferentes. Como a Rede dos Cidadãos pode ser usada por qualquer cidadão brasileiro, ela precisa levar em consideração as enormes diferenças socioeconômicas, culturais, geográficas, de conhecimento e acesso à tecnologia. Os indicadores sociais apresentados pelo Comitê de Entidades no Combate à Fome e pela Vida (COEP ) mostra que em 2003, 53 milhões de brasileiros, ou $34 \%$ da população, vivia abaixo do nível de pobreza. Ademais, cerca de 33 milhões de brasileiros, ou $26 \%$ da população, são analfabetos funcionais, o que significa que têm ao menos quinze anos de idade e menos de quatro anos de educação formal. Além disso, o recenseamento de 2000 revelou que 24,5 milhões de brasileiros, ou 14,5\% da população, apresentam deficiências . Esse aspecto está relacionado a um dos grandes desafios atuais na pesquisa da ciência da computação no Brasil, nos anos 2006 a 2016, conforme indicado pelo quarto desafio da Sociedade Brasileira de Computação: "Acesso universal e participativo ao conhecimento, para todos os cidadãos brasileiros" (SBC, 2006).

Portanto, devem ser pesquisados métodos de projeto que gerem interfaces alternativas, para garantir que todos os cidadãos brasileiros possam acessar e participar desta rede. O uso de interfaces flexíveis foi salientado na literatura como uma alternativa ao projeto de interfaces pessoa-computador, de acesso universal (SAVIDIS \& STEPHANIDIS, 2004). Essa alternativa é explorada em nosso contexto, para reduzir as barreiras de acesso à Rede dos Cidadãos, rumo a uma rede com acesso universal. Apesar de algumas barreiras não poderem ser completamente removidas com o uso de interfaces flexíveis (ou seja, acesso a computador e à Internet), elas podem ser atenuadas com a adaptação das interfaces pessoacomputador, conforme as pessoas, permitindo assim o acesso a um grande número de usuários diferentes, tais como os cidadãos brasileiros.

$\mathrm{Na}$ Rede dos Agentes de Saúde há problemas diferentes, porém igualmente árduos. Ao contrário dos cidadãos brasileiros, os usuários desta rede são profissionais e, geralmente, têm ao menos algum contato com a tecnologia. Não obstante, a interface também precisa ser flexível para que os usuários informem os resultados das pesquisas médicas, descrevendo precisamente os resultados em situações e contextos distintos. Ademais, a mobilidade e o suporte a dispositivos múltiplos são requisitos fundamentais desta rede.

No que diz respeito à Rede dos Pesquisadores, um dos desafios principais é o fornecimento de interfaces que ofereçam suporte à descrição dos protocolos com a formalidade necessária, sem a imposição de restrições, dificuldades, nem trabalho adicional. Em geral, os protocolos são descritos com o uso de linguagem técnica, do ponto de vista médico; mesmo assim, são descrições em linguagem natural. São usados termos para as ontologias comuns, objetivando maximizar o conhecimento compartilhado através da rede. Interfaces adequadas, que pesquisam e reutilizam pesquisas médicas fundamentadas nessas descrições ontológicas são fornecidas aos pesquisadores.

Sekeres et al. (2008) apresentou vários problemas relacionados à divulgação dos Registros das Pesquisas Médicas. Porém, eles também dão ênfase à importância das pesquisas médicas informadas às partes interessadas, incluindo pacientes $\mathrm{e}$ profissionais de saúde. Portanto, o WebInVivo tem por meta a exploração de soluções novas para interações entre pessoas e computadores, que realcem a qualidade das informações compartilhadas através das redes propostas. Neste contexto, essas interações apresentam desafios, como o fato dos usuários não se restringirem a profissionais versados no uso de computadores, os desenvolvedores não conhecerem os usuários alvo, e os artefatos não mais estarem restritos às especificações tecnológicas de uma plataforma com interação predefinida (SAVIDIS \& STEPHANIDIS, 2004).

Neste cenário as interfaces não são artefatos estáticos projetados por usuários de renome; ao contrário - devem ser adaptadas a vários contextos. Assim sendo, a plataforma proposta explora e aperfeiçoa técnicas de interfaces sensíveis ao 
contexto (context-aware) (MORAN \& DOURISH, 2001). As aplicações sensíveis ao contexto coletam e usam informações sobre um contexto no qual a prestação de serviços é adequada a eventos, tempo, local e pessoas específicas. No WebInVivo o contexto não se restringe aos aspectos físicos da interação; ele também considera os aspectos social, pragmático e semântico. Os aspectos semânticos denotam o significado das informações compartilhadas, enquanto os aspectos pragmáticos tratam a intenção de uso e os aspectos sociais se referem às convenções que delineiam a interação. Cremos que é possível melhorar a qualidade da interação através de análise, entendimento e simulação de situações de flexibilidade na interface do usuário. A semiótica fornece os princípios teóricos e metodológicos desta tarefa.
As normas são um conceito fundamental que nos ajuda a entender intenções, comunicações, conversas, negociações, crenças, expectativas, compromissos, contratos, leis, cultura, e também os negócios (LIU, 2000) em termos semióticos. Uma simulação fundamentada em normas é proposta como uma forma de investigar a personalização da interface no contexto do uso. Os especialistas em domínio e usuários fornecem e fazem a manutenção das normas usadas na simulação, objetivando a personalização da interface.

A Figura 5 mostra o diagrama global de uma arquitetura concebida para permitir flexibilidade em aplicações na Internet. Esta arquitetura possui dois componentes centrais: o NBIC (NormBased Interface Configurator - Configurador de Interface Fundamentado em Norma) e o ICE

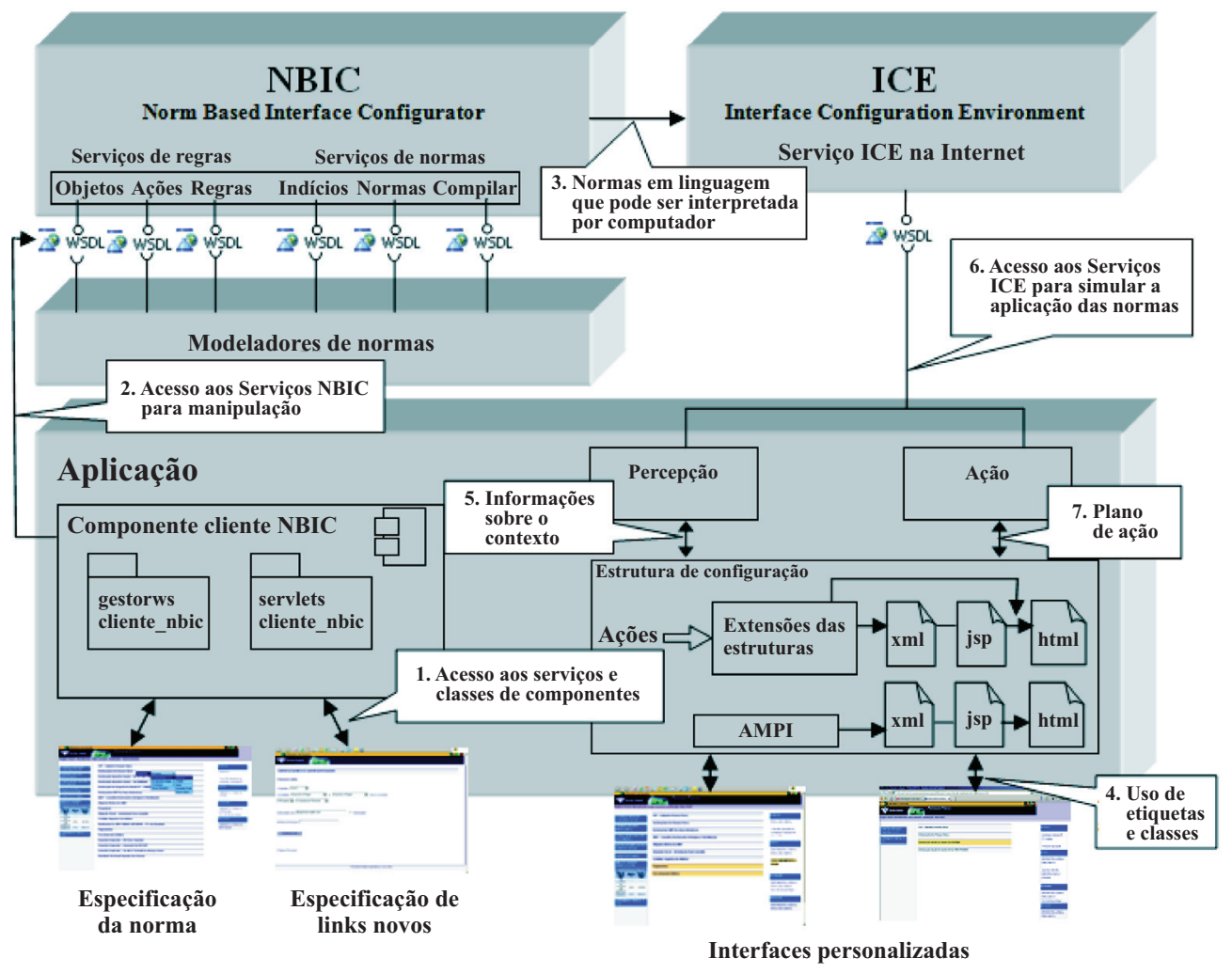

Figura 5 - arquitetura para permitir flexibilidade em aplicações via internet.

(Interface Configuration Environment - Ambiente de Configuração de Interface). A arquitetura como um todo está embasada no paradigma orientado a serviço, e na tecnologia de serviços Web. Nesta arquitetura, o NBIC fornece serviços na Internet para manipular normas e regras (associadas a uma ontologia) e compilá-las. O ICE possui serviços de manutenção das informações sobre o contexto e para a interpretação das normas (que descrevem o contexto de uso) em tempo de execução. As próprias alterações são feitas pela Tailoring Framework, que recebe um plano de ação decorrente da interpretação das normas e altera a interface com o uso de páginas dinâmicas. Propomos a adaptação e a expansão desta arquitetura para o fornecimento de interfaces adaptadas a cada rede social. O objetivo é a especificação de normas relacionadas aos recursos de cada rede social, além das normas 
fundamentadas no contexto e nas preferências individuais. As etapas a seguir podem ser seguidas para a realização da adaptação (conforme a Figura 5):

1. Os Administradores ou Especialistas em Domínio acessam os modeladores de normas para especificar as normas. Exemplo: "Se um Pesquisador acessar a Rede Social, então serão usadas as opções de busca avançada, que usam parâmetros do vocabulário do domínio do Pesquisador (da área do Pesquisador).”

2. Esta norma está armazenada no NBIC

3. A norma é traduzida para uma linguagem que pode ser interpretada por computador

4. A interface tem etiquetas específicas que assinalam pontos em que a página pode ser alterada

5. O mecanismo de percepção informa o contexto de uso. Exemplo: "O usuário é um engenheiro biológico que usa um dispositivo móvel para acessar o sistema."

6. O ICE dispara as normas e infere um plano de ação. O plano de ação é um conjunto de alterações na interface do usuário

7. O plano de ação é interpretado pelo mecanismo de ação. Exemplo: "Habilitar um botão, alterar o tamanho de divisão de uma interface e incluir um código novo de um arquivo em Extensible Markup Language (XML)".

\section{A camada lógica da aplicação}

A camada Lógica da Aplicação oferece diversos serviços de habilitação de cooperação, comunicações, além de compartilhamento de conhecimento e interpretação entre os usuários das redes sociais. As pesquisas médicas exigem a especificação do protocolo, mapeamento do protocolo, reuso do fluxo de trabalho, e constatação e composição do serviço.

\section{Constatação e especificação do protocolo}

Os pesquisadores e agentes de saúde realizam experiências conforme as especificações do protocolo. Portanto, um Gestor do Protocolo será desenvolvido para permitir a constatação e especificação dos protocolos. Essa ferramenta permitirá o desenvolvimento completo de protocolos novos ou a especialização dos que já existem. A linguagem de especificação do protocolo será fundamentada no BPMN para permitir o seu mapeamento segundo a notação BPMN. O Gestor do Protocolo oferecerá suporte ao link, para os elementos das especificações e ontologias do protocolo. Isso leva à especificação aprimorada pela semântica, que é importante para a especialização dos protocolos existentes, uma vez que permite considerar os aspectos semânticos das especificações do protocolo na descoberta de protocolos existentes.

\section{Mapeamento do protocolo para o fluxo de trabalho}

Os protocolos são mapeados como fluxos de trabalho para que possam ser executados e controlados pela plataforma.

Em virtude da complexidade envolvida na concepção dos fluxos de trabalho, é importante habilitar o reuso dos mesmos. $\mathrm{O}$ reuso minimiza os erros e diminui o custo, uma vez que o fluxo de trabalho pode ser elaborado a partir de um fluxo de trabalho existente, que já foi projetado e testado por especialistas. Um fluxo de trabalho bem construído pode ser compartilhado por especialistas que desejam realizar experiências similares (ROURE et al., 2008). A confiança no uso dos fluxos de trabalho pode ser aumentada se houver permissão para que os usuários das redes sociais recomendem os fluxos de trabalho e vejam a reputação dos mesmos nas redes.

Um meio de conseguir o reuso dos fluxos de trabalho é a criação de modelos genéricos a partir dos quais fluxos de trabalho específicos possam ser exemplificados. No entanto, em vez de usar apenas modelos, a nossa abordagem está embasada em uma técnica mais sistemática que aprimora os benefícios e custos globais. As abordagens de linha de produto e fábrica de software fornecem, especificamente, conceitos e mecanismos à unidade do setor de engenharia, o que facilita o projeto de fluxos de trabalho fundamentados em modelos de características. Assim, fluxos de trabalho específicos podem ser gerados através da configuração de fluxos de trabalho genéricos. A nossa meta é aplicar esta técnica na modelagem de partes comuns e elementos distintos dos fluxos de trabalho, para permitir a configuração dos mesmos nos casos específicos.

Apesar desta abordagem estar disponível para uso isolado, e incluir um conjunto de ferramentas que lhe fornecem suporte automático, ela ainda precisa ser integrada aos outros componentes. O conjunto de ferramentas inclui ferramentas que oferecem suporte: à geração e configuração de modelos de características e à 
exportação de arquivos XML; à geração dos modelos de fluxo de trabalho especificados nas linguagens WSDL e WS-BPEL, com base nesses modelos de características; e à exemplificação de diversos fluxos de trabalho, com base nas configurações do modelo de características.

\section{Composição de serviços}

A nossa abordagem considera o fluxo de trabalho uma composição de serviços. Para permitir a composição e a execução dos serviços, a plataforma proposta fornece um mecanismo de composição de serviços e um broker (intermediador) (nomes dos autores retirados, 2008). O mecanismo de composição de serviços usa o broker para selecionar as implementações de serviço em um registro, conforme as funcionalidades do serviço, descrições semânticas e políticas de Qualidade do Serviço (QoS) (VEDAMUTHU et al., 2007).

A composição de serviços enfoca a combinação da semântica dos serviços na Internet e dos registros dos serviços, como por exemplo o Universal Description, Discovery, and Integration - UDDI (2004), para oferecer suporte à descoberta dinâmica de serviços. A Web Ontology Language for Services - OWL-S (2004) é, por exemplo, uma linguagem de serviços semânticos da Internet que usa ontologia avançada. A OWL-S oferece constatação e raciocínio lógico no modelo do perfil de serviços, além de ontologia de processo no modelo de processo.

Resumindo, propomos três tipos de composição de serviços semânticos na Internet para a pesquisa médica: fluxo de trabalho, ontologia e privacidade. Composição de serviços baseada no fluxo de trabalho. A composição de serviços pode ser obtida com o uso da semântica do processo. Se, por exemplo, a descrição do processo semântico for publicada no registro do serviço, o broker poderá descobrir uma relação entre os modelos genéricos e os processos executáveis. Usamos, especificamente, um perfil de processo semântico como descrição semântica das atividades do processo relacionadas a um serviço na Internet. Ele apresenta as descrições de Entrada, Saída, Precondições e Efeitos (IOPE - Input, Output, Precondition and Effect) para as atividades do processo. Alem disso, o perfil dos processos semânticos pode usar uma ontologia específica do domínio como parâmetro para a descrição dos requisitos da atividade. Quando os requisitos de um processo se originam do solicitante do serviço, o broker recupera o modelo genérico e os perfis do processo semântico relacionados a ele. Depois, os perfis do processo são enviados ao Matchmaker, que encontrará serviços relevantes. Portanto, apesar dos pesquisadores médicos poderem usar fluxos de trabalho distintos, eles poderão publicar esses fluxos de trabalho como serviços e compor os serviços resultantes, usando um modelo de fluxo de trabalho genérico.

Composição de serviços fundamentada em ontologia. As ontologias são uma forma semântica de representação de conhecimento, consagrada na pesquisa médica. São usadas na representação de modelos de dados que têm por objetivo a operação conjunta de informações médicas. No entanto, o mapeamento direto das ontologias, especificamente das ontologias locais que representam modelos de dados de aplicações, é complexo. Por isso, apresentamos uma abordagem de referência fundamentada em ontologia, que usa uma combinação de serviços na Internet. Nesta abordagem, cada aplicação pode publicar as próprias ontologias como serviços na Internet, e as outras aplicações ou serviços podem recuperar esses modelos de dados e encontrar a correspondência entre as ontologias locais, através do casamento de ontologias de referência. Esta abordagem pode aumentar o número de composições de serviço possíveis, porque mesmo que as aplicações tenham modelos de dados distintos, ainda poderão operar em conjunto, se for encontrada alguma correspondência entre as ontologias das mesmas. Dessa forma, os dados gerados por aplicações distintas, em decorrência de sua execução, podem ser integrados para completar uma composição de serviços. Isso é alcançado considerando-se as informações semânticas oferecidas pela ontologia de referência, que permite o mapeamento entre os modelos de dados representados por ontologias diferentes. Portanto, a integração dos dados na pesquisa médica é feita através da composição de serviços na Internet semântica.

Composição de serviços fundamentada na privacidade. O uso de características não funcionais para a composição de serviços é valioso na pesquisa médica. A privacidade, por exemplo, é um aspecto importante no contexto deste projeto. É necessário levar em consideração os recursos do serviço e as exigências do consumidor ao incluir a privacidade na composição dos serviços. Nesta abordagem, os serviços têm de ter políticas de privacidade. Essas políticas definem como os serviços protegem a privacidade dos consumidores do serviço. No entanto, os consumidores precisam especificar as políticas que descrevem as suas preferências quando à privacidade.

Para especificar as políticas de privacidade, pode-se usar a Web Services Policy Framework - WS-Policy (VEDAMUTHU et al., 2007), que é um padrão de especificação de políticas para serviços na Internet. 
Ela oferece um modelo de política para a definição de vários tipos de política. É necessário definir, especificamente, um vocabulário de privacidade. Esse vocabulário e o modelo da política podem ser usados para a definição das políticas de privacidade, que incluem elementos como: collector, what, purpose, retention e recipient.

O primeiro desses elementos, o collector, permite que o provedor de serviços defina o nome da organização ou parte que fará a coleta dos dados. O consumidor do serviços, por outro lado, usa o elemento collector para definir quem terá permissão para coletar os seus dados. O segundo elemento, definido como what, permite que provedor e consumidor delineiem quais dados querem colocar e fornecer, respectivamente. O terceiro elemento é purpose, que permite ao provedor descrever os motivos para a coleta dos dados. Por outro lado, o consumidor usa o elemento purpose para descrever os motivos para os quais permite que as suas informações sejam coletadas. O quarto elemento, retention, permite que as duas partes determinem o intervalo de tempo durante o qual querem que os dados sejam armazenados. O quinto e último elemento é recipient, que possibilita ao provedor de serviços listar todas as partes a que poderia passar as informações que coletou. Juntos, os cinco elementos de privacidade criam uma regra única de privacidade; a política de privacidade pode consistir em uma a muitas regras.

A Figura 6 exemplifica uma política de privacidade de serviço no WS-Policy. A política indica que todos os provedores envolvidos na execução do serviço (Linha 06) podem usar todos os dados fornecidos ao serviço (Linha 04) para aprimoramento, avaliação ou revisão dos mesmos (Linha 05).

Por isso, a política de cada serviço componente e as preferências de um consumidor

\begin{tabular}{|cc|}
\hline 01 & $<$ Política $>$ \\
\hline 02 & $<$ ExatamenteUm $>$ \\
\hline 03 & $<$ Todos $>$ \\
\hline 04 & $<$ ParâmetrosDeEntrada $>$ \\
\hline 05 & $<$ Desenvolver $/>$ \\
\hline 06 & $<$ Executores $/>$ \\
\hline 07 & $</$ ParâmetrosDeEntrada $>$ \\
\hline 08 & $</$ Todos $>$ \\
\hline 09 & $</$ ExatamenteUm $>$ \\
\hline 10 & $</$ Política $>$ \\
\hline
\end{tabular}

Figura 6 - exemplo de uma política de privacidade podem ser casadas para construir uma composição de serviços no nível privacidade.

\section{Camada de recursos e conteúdo}

No que diz respeito à semântica, as ontologias oferecem suporte às camadas da Interface Pessoa-Computador e da Lógica da Aplicação. Uma ontologia de domínio pode, por exemplo, fazer parte da interface da Rede do Pesquisador, fornecendo vocabulário especializado e o reuso de thesaurus UMLS (Unified Medical Language System) (UMLS, 2008). Neste caso, as ontologias serão desenvolvidas ou reusadas e adaptadas.

Quandoumpesquisadorinsereinformações, abrindo uma nova pesquisa médica e informando o nome da doença e da experiência, uma janela popup com informações pode notificar o pesquisador sobre experiências no mesmo domínio. Assim, os serviços que oferecem suporte ao desenvolvimento do protocolo, alertando o pesquisador sobre serviços na Internet e fluxos de trabalho disponíveis, farão parte desta camada. Esses serviços permitirão o possível reuso do conhecimento existente nas redes, o que será decorrente de um serviço de busca e classificação que opera em segundo plano.

$\mathrm{O}$ processo de pesquisa médica consiste em uma sequência de etapas, que pode ser descrita como uma cadeia, e contém vocabulário específico. $\mathrm{O}$ resultado de uma etapa pode ser a entrada para outra etapa ou para etapas paralelas. Este método irá gerar a descrição do processo ontológico, com parâmetros de entrada e saída, além de eventos, que podem ser articulados a outros. Isso cria as relações que também são descritas pelas ontologias.

As ontologias também são necessárias para a conexão de redes sociais diferentes. A equivalência semântica pode ser usada na constatação de vocabulários distintos com o mesmo significado. Sensores semânticos, que incluem sinônimos, grupos de palavras e palavras já conhecidas, podem interconectar as redes, enviando um alerta a elas. As ontologias também serão concebidas para adicionar informações às informações, como na etiquetagem e recomendação de documentos ou fluxos de trabalho.

\section{Trabalho relacionado}

As iniciativas de Trabalho em Colaboração com Suporte de Computador (CSCW - ComputerSupported Collaborative Work) para suporte à colaboração e interação científica não são novas, apesar do uso desses sistemas em grande escala ainda enfrentar várias barreiras entre os pesquisadores. $\mathrm{Na}$ pesquisa médica, ao ceticismo são somados a complexidade e os requisitos 
de privacidade durante o desenvolvimento da pesquisa. Primeiro, os pesquisadores CSCW enfocaram a modelagem "trabalho administrativo" para o aperfeiçoamento das colaborações durante a realização dos processos organizacionais (LUBICH, 1995). Em organizações comerciais, as aplicações de rede social são projetadas para aperfeiçoar as interações não formalizadas que não contam com o suporte dos sistemas do fluxo de trabalho. $\mathrm{Na}$ pesquisa médica, seria útil se um modelo de fluxo de trabalho, ou parte dele, pudesse sem tratado como se as comunicações e interações estivessem nas aplicações "regulares" da rede social.

Tal como o WebInVivo, outras iniciativas revolucionárias foram desenvolvidas para promover e facilitar a colaboração entre os pesquisadores. Um exemplo bom é o MyExperiment (ROURE et al., 2008) que expandiu o uso dos sistemas de fluxo de trabalho e redes sociais para o processo de desenvolvimento de pesquisa. As tarefas do "espectro" a que o MyExperiment oferece suporte incluem protocolos e procedimentos bem estruturados, além das tarefas criadas durante o processo de desenvolvimento da pesquisa.

A rede social MyExperiment (ROURE et al., 2008) compartilha fluxos de trabalho científicos usando a arquitetura Web 2.0. A pesquisa e exposição do fluxo de trabalho estão fundamentadas em técnicas da Web 2.0, como etiquetas, recomendações, citações, revisões e discussões em blog. Além disso, o uso de wikis na área biomédica está se tornando muito difundido, o que facilita o compartilhamento de protocolos. Um exemplo disso é o OpenWetWare (OPENWETWARE, 2008), que não foi desenvolvido por especialistas na Web 2.0, além do paradigma orientado ao serviço. O WebInVivo também usa técnicas da Web 2.0 e o paradigma orientado ao serviço, porém amplia essas técnicas com o uso de ontologias de domínio dentro da arquitetura Web 2.0

A semântica Web, que define linguagens, modelos de inferência e serviços na Internet, ajuda a caracterização da informação, com base em uma ontologia de etiquetagem, que permite a filtragem e a recomendação de conteúdo ((GRUBER, 2007). O UMLS (2008) é outro exemplo de compartilhamento de conhecimento embasado em ontologias de domínio e ferramentas de conhecimento, como o thesaurus, que existe no ambiente da Internet. Este trabalho propõe uma abordagem de colaboração fundamentada na ontologia. O WebInVivo emprega essa abordagem, junto com a tecnologia de fluxo de trabalho e o paradigma orientado ao serviço para fornecer uma estrutura abrangente à pesquisa médica.
Um dos primeiros projetos que usou a modelagem de protocolos em sistemas computacionais foi o Arden (CLAYTON et al., 1989). Outras fases do ciclo de vida, que incluem o teste e a execução do protocolo, foram estudadas em GLIF (PELEG et al., 2004) e Gaston (CLERCQ et al., 2004). Apesar do WebInVivo tratar o ciclo de vida do protocolo, ele está fundamentado em tecnologias mais avançadas, como o paradigma orientado ao serviço e os recursos da Web 2.0 para colaboração e compartilhamento. De fato, a orientação rumo a uma arquitetura direcionada a serviços já fora definida por agências europeias (IMI, 2008) e norte-americanas (KAWAMOTO \& LOBAC, 2007).

\section{Benefícios da tecnologias de internet}

A arquitetura proposta, fundamentada em tecnologias da Internet, pode oferecer um ambiente de pesquisa com colaboração, além da possibilidade de compartilhamento de conhecimento e ativos entre os usuários de redes sociais.

O WebInVivo pode contribuir com a pesquisa médica. Primeiro, o reuso do protocolo, reduz tempo e custo na produção de fármacos novos. Ademais, com o uso da tecnologia de fluxo de trabalho os atores responsáveis podem ser notificados sobre prazos e outras atividades relacionadas à execução do protocolo. Além disso, há uma redução do erro, porque fluxos de trabalho bem definidos podem ser compartilhados para a realização de experiências similares. Por fim, há mais controle e confiabilidade, com os pacientes participando das pesquisas médicas através de redes sociais.

Há uma série de contribuições relacionadas às redes sociais, uma das quais é a criação e disseminação do conhecimento através de redes sociais interconectadas de forma seletiva. A arquitetura também gera a promoção da população afetada por doenças, e a detecção de problemas e de áreas de risco através de relatórios de agentes de saúde e da população. Isso faz com que médicos e pesquisadores ampliem e reusem os protocolos existentes. Por fim, leva à colaboração entre grupos de pesquisadores e à promoção de cursos de e-learning.

O WebInVivo oferece contribuições adicionais que incluem interfaces que se adaptam a vários contextos. Através da interface, os pesquisadores podem descrever os protocolos com a formalidade necessária, sem a imposição de restrições nem de trabalho adicional durante esta tarefa. Ele também amplia o paradigma orientado 
ao serviço às aplicações de rede social e ao ambiente de pesquisa em colaboração, com o uso de técnicas de semântica da Internet. Como a pesquisa médica requer a especificação do protocolo, mapeamento do protocolo, reuso do fluxo de trabalho e constatação e composição do serviço, a plataforma permite cooperação, comunicação, e compartilhamento de conhecimento e interpretação entre os usuários das redes sociais. A contribuição final envolve ontologias que oferecem suporte à interoperabilidade entre redes sociais distintas.

\section{Conclusões e trabalho futuro}

A plataforma WebInVivo tem por objetivo oferecer suporte à pesquisa médica e ao compartilhamento de protocolos para a realização de experiências médicas. Algumas contribuições importantes de TI estarão relacionadas a mecanismos que mapeiam protocolos para fluxos de trabalho, reuso de fluxos de trabalho e composição de atividades de fluxo de trabalho implementadas por serviços na Internet. Além disso, as redes sociais simplificam a elaboração e o compartilhamento de conhecimento sobre a prevenção e o tratamento, de forma acessível aos diversos segmentos da sociedade brasileira.

Neste artigo, identificamos os requisitos da pesquisa médica e propomos uma arquitetura fundamentada em serviços, para um ambiente de colaboração que permite o compartilhamento de informações através de redes sociais.

O WebInVivo é uma proposta cuja implementação incluiráaintegração decomponentes existentes que implementem funcionalidades à Camada Lógica da Aplicação. Outras camadas do WebInVivo foram propostas para oferecer suporte à especialização da camada Lógica da Aplicação no âmbito da pesquisa médica. Os componentes que têm de ser implementados para que todas as camadas possam desempenhar as funcionalidades requeridas foram descritos neste artigo.

O trabalho futuro inclui a ampliação da plataforma para permitir o acesso de pessoas às redes sociais através de dispositivos moveis, como assistentes digitais pessoais e telefones celulares. Para atender esse requisito, a infraestrutura deve ser adaptada para fornecer a possibilidade de configuração e personalização dos diversos tipos de aplicações e dispositivos móveis, além da conscientização quanto aos recursos e adaptabilidade do tempo de execução para a gestão das alterações dinâmicas do ambiente. Especificamente, as técnicas de middleware para dispositivos móveis devem ser avaliadas para que essas metas sejam alcançadas (nomes dos autores retirados, 2007).

Este trabalho foi realizado por universidades e um centro de pesquisa, sendo financiado por agências canadenses e brasileiras, na forma de bolsas de estudo de graduação.

\section{Notas}

1. http://www.coepbrasil.org.br/

2. http://portal.mec.gov.br

3. http://mj.gov.br

\section{Referências bibliográficas}

ALLISON, D. S.; El YAMANY, H.; CAPRETZ, M. A. M. Metamodel for Privacy Policies within SOA. Proceedings Of The 5th International Workshop on Software Engineering for Secure Systems (SESS'09), Vancouver, Canada, May 19, 2009.

ALONSO, G.; CASATI, F.; KUNO, H.; MACHIRAJU, V. Web services: Concepts, Architectures, and Applications. Springer Verlag, Berlin, 2004.

ALVES, A. et al. Web Services Business Process Execution Language Version 2.0 Specification. OASIS, Apr. 2007. http://docs.oasis-open.org/ wsbpel/2.0/OS/wsbpel-v2.0-OS.html, accessed in 01/2008.

BERNERS-LEE, T. Weaving the Web: The Past, Present and Future of the World Wide Web by its Inventor. Orion Business Books. http://www. w3.org/People/ Berners-Lee/Weaving, 1999.

BERNERS-LEE, T.; HENDLER, J.; LASSILA, O. The Semantic Web. Scientific American, 284(5):3543, 2001 .

BONACIN, R.; BARANAUSKAS, M. C. C.; dos SANTOS, T. M. A semiotic-based framework for the development of tailorable Web applications. Lecture Notes in Computer Science, 4553:859868, 2007.

BONACIN, R.; BARANAUSKAS, M. C. C.; LIU, K.; SUN, L. Norms-based simulation for personalized service provision. Semiotica, Journal of the International Association for Semiotic Studies, 175(1/4):403-428, 2009.

CALlAN, B.; GILlESPIE, I. The Path to New Medicines. Nature, 449(7159):164-5, 2007.

CLAYTON, P.D.; PRYOR, T.A.; WIGERTZ, O.B.; HRIPCSAK, G. Issues and structures for sharing knowledge among decision-making systems: The 1989 Arden Homestead Retreat. In: Kingsland LC, editor. In Proceedings of the Thirteenth Annual 
Symposium on Computer Applications in Medical Care, pages 1 16-21. IEEE Computer Society Press, 1989.

CLERCQ, P.; HASMAN, A. Experiences with the Development, Implementation and Evaluation of Automated Decision Support Systems. Stud Health Technol Inform, 2004, http://www. openclinical. org/gmm_gaston.html, accessed on 10/2008.

DUMAS M.; AALST, W., HOFSTEDE A. (eds) Process-Aware Information Systems: Bridging People and Software Through Process Technology. Wiley InterScience, Hoboken, NJ, US, 2005.

FANTINATO, D. Z. G.; TOLEDO, M. B. F. GIMENES, I. M. S. WS-Contract Establishment with QoS: an Approach Based on Feature Modeling. Int. J. Cooperative Inf. Syst., 17(3):373-407, 2008.

FANTINATO, M.; GIMENES, I. M. S; TOLEDO, M. B. F. A Product Line for Business Process Management. In Proceedings of the 12th International Software Product Line Conference, Limerick, Ireland, 2008.

GARCIA, D. Z. G.; TOLEDO, M. B. F. A Privacy Framework for Web Services. In Proceeding of the Web2Touch, IEEE 11 th International Conference on Computational Science and Engineering, 2008.

GRUBER, T. Ontology of Folksonomy: A Mashup of Apples and Oranges. International Journal on Semantic Web \& Information Systems, 3(2), 2007.

KAWAMOTO, K.; LOBAC, D. Proposal for Fulfilling Strategic Objectives of the U.S. Roadmap for National Action on Decision Support through a Service-oriented Architecture Leveraging HL7 Services. J Am Med Inform Asso,. Mar-Apr; 14(2), 2007.

LIU, K. Semiotics in information systems engineering. Cambridge: Cambridge University Press, 2000.

LUBICH, H. TOWARDS. a CSCW Framework for Scientific Cooperation in Europe (Lecture Notes in Computer Science). Berlin: Springer-Verlarg, 1995.

MORAN, T; DOURISH, P. Introduction to the special issue on Context-Aware Computing. Human-Computer Interaction, 2(3), 2001.

OpenWetWare, http://openwetware.org/wiki/ Main Page, accessed in 10/2008.

O'REILLY, T. What is Web 2.0: Design Patterns and Business Models for the Next Generation of
Software, accessed in January 2009. http://www. oreillynet.com/go/web2, 2005.

OWL-S Web Ontology Language for Web services http://www.w3.org/Submission /OWL-S/, 2004.

PAPAZOGLOU, M.P.; VAN DEN HEUVEL, W.J. Service Oriented Architectures: Approaches, Technologies and Research Issues. VLDB Journal, 16(3):389-415, 2007.

PELEG, M. et al. The InterMed Approach to Sharable Computer-Interpretable Guidelines: a Review. J Am Med Inform Assoc., Jan-Feb; 11 (1), 2004.

PINELLE, D., GUTWIN C., Loose Coupling and Healthcare Organizations: Deployment Strategies for Groupware. Journal of Computer Supported Cooperative Work 15:537-572. 2006.

ROCHA, T.; ARNTSEN, A.-B.; EIDSVIK, A. K.; TOLEDO, M. B. F; KARLSEN, R.. Promoting Levels of Openness on Component-Based Adaptable Middleware. In Proceedings of the 6th Workshop on Adaptive and Reflective Middleware, Newport Beach-CA, United States, 2007.

ROURE, D.; GOBle, G.; STEVENS, R. The Design and Realisation of the myExperiment Virtual Research Environment for Social Sharing of Workflows, http://www.myexperiment.org/, accessed in 10/2008.

SAVIDIS, A.; STEPHANIDIS, C. Unified user interface development: the software engineering of universally accessible interactions. Universal Access in the Information Society 3:165-193. 2004.

SBC. Grand Challengers in Computer Science, 2006 - 2016. http://www.sbc.org.br/, accessed in 06/2009.

SEKERES, M. et al. Poor Reporting of Scientific Leadership Information in Clinical Trial Registers. PLoS ONE, 3(2) 2008.

SORIANO, J. et al. Enhancing User-Service Interaction Through a Global User-Centric Approach to SOA. In Proceedings of the Fourth International Conference on Networking and Services, 2008.

TAYLOR, I.; DEELMAN, E.; GANNON, D.; SHIELD, M. (Eds.). Workflows for e-Science. Springer, 2006.

IMI - The Innovative Medicines Initiative (IMI) Research Agenda, http://imi.europa.eu/ docs/ imigb-006v2-15022008-research-agenda_en.pdf, accessed in 10/2008. 
TOLEDO, M. B. F; NABUCO, O.; RODRIGUES, M.; GARCIA, D. Z.; CAPRETZ, M. A. M.; FANTINATO, M.; GIMENES, I.; BONACIN, R.; GUERRA, A.; ROCHA, T.; VIANA, L. A SOAbased Collaborative Environment for Clinical Trials on Neglected Diseases. In Proceedings of 4th International IEEE Workshop on Service Oriented Architectures in Converging Networked Environments, in conjunction with The IEEE 23rd International Conference on Advanced Information Networking and Applications (AINA-09) Bradford, UK, May 26-29, 2009.

TOLEDO, M. B. F; NABUCO, O.; RODRIGUES, M.; CAPRETZ, M.; FANTINATO, M.; GIMENES, I.; BONACIN, R.; GUERRA, A.; ROCHA, T.; JINO, M. Gestão do Conhecimento e da Colaboração em Pesquisa Clínica, In Proceedings of II Seminário sobre Grandes Desafios da Computação no Brasil, Manaus, Brasil, March 3-4, 2009.

UDDI Universal Description, Discovery and Integration, Version 3.0.2, 2004.

UMLS - Unified Medical Language System http:// kswebp 1.nlm.nih.gov/uPortal/frame.jsp?myvtframe $=$ http://www.nlm.nih.gov/ research/umls/ documentation.html, 2008.

VEDAMUTHU, A. S. et al. Web Services Policy 1.5 - Framework. W3C, Sep. 2007. http://www.w3.org/ TR/2007/REC-ws-policy-20070904/, accessed on 06/2009.

WSDL - Web Services Description Language. Version 2.0, W3C Recommendation, June 2007. http://www.w3.org/TR/wsdl20/

\section{Sobre os autores}

\section{Miriam A. M. Capretz}

A Dra. Miriam A. M. Capretz é Professora Adjunta e Catedrática Adjunta - Graduada pelo Departamento de Engenharia Elétrica e de Computação da Universidade de Western Ontario, Canadá. Antes de entrar para a Universidade de Western Ontario foi Professora Adjunta do Laboratório de Engenharia de Software, da Universidade de Aizu, Japão. A Dra. Miriam Capretz concluiu a graduação e obteve o grau de mestre pela UNICAMP, Brasil, e o PhD pela Universidade Durham, Reino Unido. Ela participou da organização de vários workshops e simpósios, além de atuar como membro do comitê programático em diversas conferências internacionais. Foi Diretora Adjunta de Programa do IEEE Workshop Web2Touch 2009 - experiência de vida através da Internet (W2T) em 2008 e 2009, além de ser Diretora de Programa do Simpósio IEEE sobre Computação Orientada a Serviços Humanos e Socioculturais em 2009. Trabalha na área de engenharia de software há mais de 25 anos. Atualmente, os seus interesses em pesquisa incluem a arquitetura orientada a serviços, ontologia e integração semântica, gestão de processos de negócios, segurança de software e computação em grade.

\section{Maria Beatriz Felgar de Toledo}

Maria Beatriz Felgar de Toledo é professora adjunta do Instituto de Computação da Universidade de Campinas (Unicamp), Brasil. Graduou-se em Ciência da Computação pela Unicamp; doutourou-se pela Universidade Lancaster, Reino Unido (1992). Organizou as três primeiras edições do Workshop Brasileiro em Gestão de Processos de Negócios, desde 2007. É membro do comitê do Simpósio Brasileiro de Sistemas de Informação; organizou o workshop IEEE Web2Touch em 2008 e 2009, e o Simpósio IEEE sobre Computação Orientada ao Serviço Humano e Sociocultural, realizado em Toronto, Canadá, em setembro de 2009. Os seus principais interesses em pesquisa são modelos de transação avançados, computação orientada a serviços, computação em grade, sistemas de gestão de processos de negócios, sistemas de base de conhecimento para pesquisa médica e herança cultural. 